(C) 2004. This manuscript version is made available under the CC-BY-NC-ND 4.0 license http://creativecommons.org/licenses/by-nc-nd/4.0/

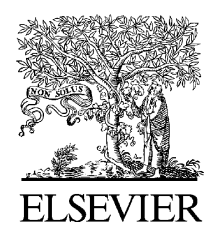

Available online at www.sciencedirect.com

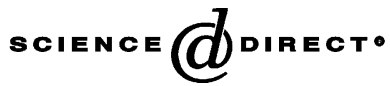

Sensors and Actuators B xxx (2005) xxx-xxx

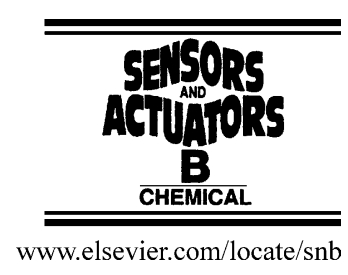

\title{
Strategies for fabrication of hydrogen peroxide sensors based on electrostatic self-assembly (ESA) method
}

\author{
Ignacio Del Villar ${ }^{\mathrm{a}}$, Ignacio R. Matias ${ }^{\mathrm{a}, *}$, Francisco J. Arregui ${ }^{\mathrm{a}}$, \\ Jesús Echeverría ${ }^{b}$, Richard O. Claus ${ }^{c}$ \\ ${ }^{a}$ Departamento de Ingeniería Eléctrica y Electrónica, Universidad Pública de Navarra, 31006 Pamplona, Spain \\ ${ }^{\mathrm{b}}$ Departamento de Química, Aplicada Universidad Pública de Navarra, 31006 Pamplona, Spain \\ ${ }^{c}$ Fiber and Electro-Optics Research Center, The Bradley Department of Electrical and Computer Engineering, 106 Plantation Road, \\ Virginia Polytechnic Institute and State University, Blacksburg, VA 24061, USA
}

Received 12 July 2004; received in revised form 27 October 2004; accepted 28 October 2004

\begin{abstract}
An optical fiber sensor has been fabricated for detection of $5 \mu \mathrm{M}-0.1 \mathrm{mM}$ concentrations of hydrogen peroxide with a linear response. The deposition method used is electrostatic self-assembly (ESA) of polymer cationic and anionic layers. Prussian Blue (PB) has been included in the polycation layers. The optical fiber sensor is included in a reflection setup, where the measuring technique is based on the slope of the optical reflected power change caused by oxidation of Prussian White (PW) to Prussian Blue. The sensor recovers after immersion in a reductive agent and is immune against a variety of components. Measurement of hydrogen peroxide has been proved successfully in a wide range $\mathrm{pHs}$ between 3 and 9 . Some techniques have been applied in order to avoid the lost of indicator.
\end{abstract}

(C) 2004 Elsevier B.V. All rights reserved.

Keywords: Electrostatic self-assembly; Hydrogen peroxide; Optical fiber sensor; Prussian Blue

\section{Introduction}

Accurate estimation of hydrogen peroxide $\left(\mathrm{H}_{2} \mathrm{O}_{2}\right)$ is important in many fields. It is used in many industrial processes as an oxidizing, bleaching and sterilizing agent. It is also the waste product in industries such as atomic power stations. In these cases, concentration of $\mathrm{H}_{2} \mathrm{O}_{2}$ is higher than in pharmaceutical, clinical and environmental analysis, where it is the product of reactions catalyzed by a large number of oxidase enzymes. These last fields require accurate sensors that can detect low concentrations. Consequently, in this work we fabricate a sensor adequate for detecting micromolar concentrations of $\mathrm{H}_{2} \mathrm{O}_{2}$.

\footnotetext{
* Corresponding author. Tel.: +34 948 169288; fax: +34 948169720 .

E-mail addresses: ignacio.delvillar@unavarra.es (I. Del Villar), natxo@unavarra.es (I.R. Matias), parregui@unavarra.es (F.J. Arregui), jesus.echeverria@unavarra.es (J. Echeverría).
}

To this purpose, three important characteristics must be taken into account. The first one is the detection technique. Since the determination of $\mathrm{H}_{2} \mathrm{O}_{2}$ concentration has been a matter of research during the last years, and it still continues today, there are a wide variety of techniques. Apart from titration [1], there are two main groups: electrochemical detection [2-10] and spectroscopy [11-21]. Both of them are adequate for detection of micromolar range concentrations. The latter avoids the application of a voltage and the necessity of smart membranes to protect the electrodes. But, the main reason for the selection of spectroscopy is the idea of deposing the sensitive structure in an optical system where the substrate is optical fiber. The advantages of optical fiber are well known: immunity against electromagnetic interferences, capability of multiplexing several sensors, low losses and so forth. Among the variants of spectroscopy: chemiluminescence [11,12], spectrofluorometry [13-16] and spectrophotometry [10,17-21]; the last one has been chosen. The substance to detect oxidizes a chromogenic hydrogen donor. 
Its refractive index changes in the transition from the reduced to the oxidized state, which leads to an absorbance change that can be detected with a simple optical system. This phenomenon has been studied in the oxidation of Prussian White (PW) to Prussian Blue (PB) $[10,20,21]$. An important advantage of the $\mathrm{PW} / \mathrm{PB}$ system compared with other sensors $[2,4-7,11,14,15,18]$, is that no enzyme is needed in the sensitive structure. This permits to save costs. Enzymes such as horseradish peroxidase (HRP) are still expensive nowadays. Anyway, there are some less expensive mimics that avoid the usage of HRP $[11,15]$. However, most of them are organic, which reduces the lifetime of the sensor in an important way, in comparison with the sensor developed in this work, as it will be proved. Furthermore, the changes in the absorbance of PW/PB system are present in a wide range of wavelengths between 600 and $1000 \mathrm{~nm}$. This permits the usage of the first optical telecommunications window, where LED sources and photodetectors are cost effective.

The second characteristic is the deposition technique. Electrostatic self-assembly (ESA) method has been selected. It is based on the construction of molecular multilayers by the electrostatic attraction between oppositely charged polyelectrolytes in each monolayer deposited, and involves several steps [22]. It is used to build up coatings on a wide variety of substrates such as ceramics, metals, and polymers of different shapes and forms, including planar substrates, prisms, and convex and concave lenses. Fiber optic is also included as a possible substrate $[13,17,23]$, which is the one selected in this work. One advantage of this technique compared with others is the stable electrostatic attraction of the nanostructure deposited at the end of the fiber pigtail. Secondly, there is wide variety of different polymer structures where Prussian Blue can be deposited. This should permit to avoid the interference of some molecular species if an adequate structure is chosen. In addition to this, the thickness of the cavity created at the end of fiber is lower than the coherence wavelength of a LED source. This permits to avoid the necessity of an expensive laser device to monitor interferometric phenomenon caused by changes in the refractive index of the material deposited. Since the range of absorbance change of Prussian Blue is located in the first optical communications window, a simple LED source and a photodetector are adequate for the optical detection scheme.

The last characteristic is the measuring technique. This is one of the major novelties of this sensor. The detection is based on a reflection scheme where the slope of the change of absorbance of PW/PB system indicates the concentration of $\mathrm{H}_{2} \mathrm{O}_{2}$. This phenomenon is due to the diffusion time of $\mathrm{H}_{2} \mathrm{O}_{2}$ inside the polymer structure, where Prussian Blue is included. The diffusion time is dependent on the concentration of $\mathrm{H}_{2} \mathrm{O}_{2}$.

The concentrations that can be monitored with a linear response range from $5 \mu \mathrm{M}$ to $0.1 \mathrm{mM}$. The $\mathrm{pHs}$ analyzed range from 3 to 9 , which includes the physiological $\mathrm{pH}$. This wide $\mathrm{pH}$ range is possible due to the lack of an enzyme in the structure deposited at the end of fiber.

\section{Experimental}

\subsection{Reagents}

The polycation poly(allylamine hydrochloride, $\mathrm{PAH}$ ), $\mathrm{M}_{\mathrm{w}}$ 70000 , and the polyanion poly(acrylic acid, PAA), $\mathbf{M}_{\mathrm{w}}$ 30000, were obtained from Aldrich. Prussian Blue soluble $\left(\mathrm{C}_{6} \mathrm{Fe}_{2} \mathrm{KN}_{6} \cdot x \mathrm{H}_{2} \mathrm{O}\right)$ was obtained from Riedel-de Haën AG. Ascorbic acid was obtained from Panreac. Acetic and phosphate buffer solutions of $25 \mathrm{mM}$ ionic strength were fabricated in our laboratory. Hydrochloric acid $(\mathrm{HCl})$ and sodium hydroxide $(\mathrm{NaOH})$ were used to adjust the $\mathrm{pH}$ of the polymers and Prussian Blue solutions. All water was filtered through a Milipore Q plus 185 purification system.

\subsection{Instrumentation}

Power measurements were obtained using a reflection scheme where the light source is a Hewlett-Packard 9537 HFBR 1424 LED at $810 \mathrm{~nm}$ and an Ophir LaserStar photodetector detects the reflected power measurements at the first optical communications window. The $\mathrm{pH}$ of the different solutions was adjusted with a Crison GLP22 $\mathrm{pH}$ meter. In order to obtain the spectrum of Prussian Blue deposited in the polymer structure, an halogen white source and an Avantes AVS S2000 spectrometer replace the LED source and the photodetector, respectively in the reflection scheme.

\subsection{Assembly of multilayer films}

The materials used for deposition of cationic and anionic layers are PAH and PAA, respectively. The concentration of PAH is $2 \mathrm{~g} / \mathrm{l}$ and PAA $5 \mathrm{ml} / 100 \mathrm{ml}$. The method used for including Prussian Blue into the polymeric structure is premixing with the polycation [13]. In this method, the dye is mixed with a polyion with the opposite charge. Both of them will associate with the other oppositely charged polyion, leading to the construction of the structure. Some experiments where performed with conjugation technique. Prussian Blue was mixed with PAA, but changes in the signal were not as clear as with premixing. Annealing after concluding the construction of the LBL structure at the end of the fiber pigtail is another important parameter with consequences in the performance of the sensor. It has been proved that sensors must stay at $100{ }^{\circ} \mathrm{C}$ during at least $2 \mathrm{~h}$ for a good performance. On the other hand, the addition of capping bilayers at a different $\mathrm{pH}$ at the end of the structure causes an improvement in the sensitivity of the sensor.

\subsection{Measuring technique}

To perform each measure the same procedure is repeated: the sensor is immersed in a reductive agent, such as ascorbic acid solution, to reduce PB to PW. After this, the sensor is immersed in a buffer solution. When the signal is stabilized, $\mathrm{H}_{2} \mathrm{O}_{2}$ is injected in the buffer, which oxidizes PW to PB. A 
change in the reflected optical power is then produced. This process will be repeated for other measures, which indicates that it is a multiple use sensor. The response time of the sensor as a function of hydrogen peroxide concentration is exponential. To avoid this problem, another measuring technique is proposed: the quotient between two values: the difference of power between $90 \%$ and $10 \%$ of the complete signal level change, and the time elapsed between these two values. This slope leads to good linearity as a function of the concentration. That is the reason for selecting it. In Section 3 it will be referenced, for the sake of simplicity, as the slope of the reflected optical power detected by the reflection scheme.

\section{Results and discussion}

\subsection{Sensing mechanism}

A reflection scheme for the sensor is represented in Fig. 1. The light launched by a LED source is reflected by the sensing arm of a coupler and detected at the photodetector. This system is used for the purpose of controlling the growth of the structure. Bilayers of $\left[\mathrm{PAH}+\mathrm{PB}^{+} / \mathrm{PAA}^{-}\right]$are deposited at the end of fiber. This creates a nano Fabry-Perot cavity between the fiber and the external medium. As more bilayers are deposited, the reflected power describes a sinus plot explained in terms of interferometry [24]. Once the structure has been deposited, the same setup is used for detecting variations in the reflected optical power if the absorbance of the $\mathrm{PW} / \mathrm{PB}$ system is changed by the presence of $\mathrm{H}_{2} \mathrm{O}_{2}$.

\subsection{Choice of materials}

Prussian Blue has been selected based on its applicability to the detection of $\mathrm{H}_{2} \mathrm{O}_{2}$ in both electrochemical sensors [8,9] and spectroscopy $[10,20,21]$. The absorbance plot for the structure deposited at the end of fiber is strongly influenced by the presence of Prussian Blue. In [20,21], it is asserted that the maximum is located at a wavelength of $720 \mathrm{~nm}$. This is actually the maximum for this pigment in bare solution. Nonethe-

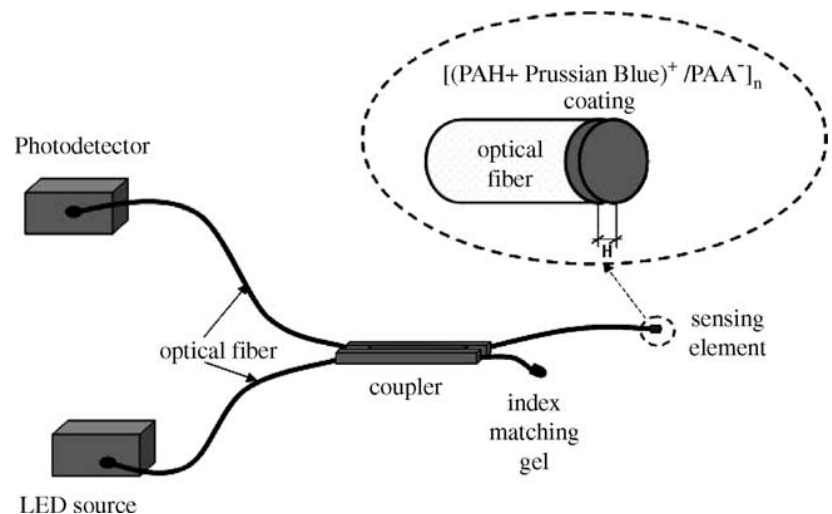

Fig. 1. Experimental setup to detect the reflected optical power by the nano Fabry-Perot cavity.

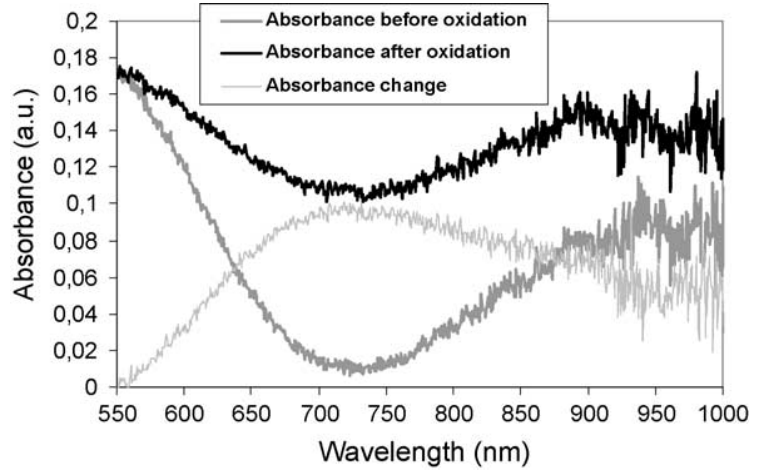

Fig. 2. Absorbance before and after oxidation, and absorbance change of $\mathrm{PAH}+\mathrm{PB} / \mathrm{PAA}$ structure with $\mathrm{H}_{2} \mathrm{O}_{2}$ at $\mathrm{pH} 7$.

less, there are some cases where this assumption is not valid. In [19], Meldola's Blue is immersed in a matrix. This causes a shift in the absorbance maximum that is characteristic of this colorant from 570 to $720 \mathrm{~nm}$. As a result, we have obtained in Fig. 2 the spectrum absorbances of both Prussian White (the reduced form of Prussian Blue) and Prussian Blue. It is also included the difference between both absorbance plots. The reflection scheme of Fig. 1 has been modified by replacing the LED source with a white light source, and the photodetector with a spectrometer. Around $720 \mathrm{~nm}$, it is located the maximum of absorbance change. Indeed, there is a wide range between 600 and $1000 \mathrm{~nm}$, where measures can be taken. Consequently, it has been demonstrated that the polymer structure where Prussian Blue is immersed causes no appreciable shift in the absorbance spectrum of the colorant in bare solution. Any LED source, in the first optical communications window or even beyond, can be applied in the reflection scheme for detection of different concentrations of $\mathrm{H}_{2} \mathrm{O}_{2}$.

\subsection{Sensors without capping bilayers}

In this section, homogeneous polymeric structures deposited at the end of fiber are analyzed. As an example, a sensor composed of 17 bilayers has been cured during $2 \mathrm{~h}$ at $100^{\circ}$. The thermal treatment avoids progressive desorption of the bilayers. However, sensitivity is an important parameter that is reduced if higher thermal treatment is performed. Consequently, a trade off must be maintained between sensitivity and robustness. Both factors cannot be improved at the same time. There is an additional trade off that optimizes the sensors response when 17 bilayers deposited. It was shown that the sensitivity of the sensors increased as the amount of Prussian Blue deposited augmented, i.e. as the number of bilayers increased. However, the stability of the structure decreased if too many bilayers were deposited. Seventeen bilayers were considered an optimum value in our experiments.

In Fig. 3, the response of the sensor against different concentrations of $\mathrm{H}_{2} \mathrm{O}_{2}$ is analyzed. Measures have been taken 1 week later than its construction, once the sensor has stabilized its sensitivity. This phenomenon is explained in terms 


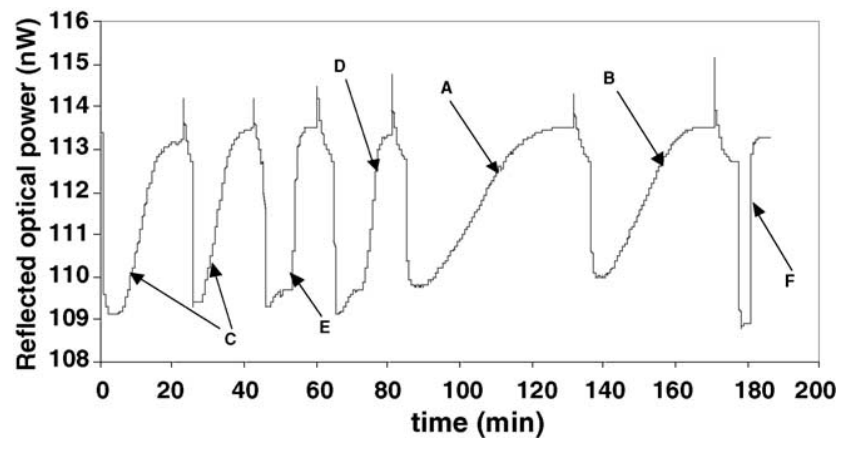

Fig. 3. Reflected optical power for different concentrations of $\mathrm{H}_{2} \mathrm{O}_{2}$ at $\mathrm{pH} 4$. $\mathrm{A}=5 \mu \mathrm{M}, \mathrm{B}=10 \mu \mathrm{M}, \mathrm{C}=20 \mu \mathrm{M}, \mathrm{D}=50 \mu \mathrm{M}, \mathrm{E}=100 \mu \mathrm{M}$ and $\mathrm{F}=500 \mu \mathrm{M}$.

of lost of the pigment. During the first measures, Prussian Blue leached from the structure until the amount of Prussian Blue inside the polymer structure was stabilized. At this point, the response of the sensor is stable and results can be reproducible. The $\mathrm{pH}$ of the solutions is 4 and the concentrations analyzed range between $5 \mu \mathrm{M}$ and $0.1 \mathrm{mM}$. It can be appreciated that the slope of the change in the reflected optical power increases, as the concentration of $\mathrm{H}_{2} \mathrm{O}_{2}$ is higher. This effect is caused by the diffusion of $\mathrm{H}_{2} \mathrm{O}_{2}$ in the polymer structure. As the concentration is higher the diffusion is faster, and the slope increases. The slope of the change of signal (see Fig. 4a) presents a linear dependence on the concentration of $\mathrm{H}_{2} \mathrm{O}_{2}$. The multiple-correlation coefficient $R^{2}$ is indicated and is very close to one. Even a wider range of $\mathrm{H}_{2} \mathrm{O}_{2}$ could be detected but the response was not linear. On the other hand, in Fig. $4 \mathrm{~b}$ the response time of the sensor for different concentrations of $\mathrm{H}_{2} \mathrm{O}_{2}$ presents an exponential shape.

To prove the durability of the sensor, in Fig. 5 the response of the same sensor 3 months after its construction and after many measures at different $\mathrm{pHs}$ is analyzed. In this case, the response of the sensor for the same concentration of $10 \mu \mathrm{M}$ at pHs 3, 5, 7 and 9 is analyzed. It was checked that the structure desorbs for a $\mathrm{pH} 11$. The conclusion is that there is a dependence of the response on the $\mathrm{pH}$ (see upper right corner of Fig. 5), but not as high as for other sensors where

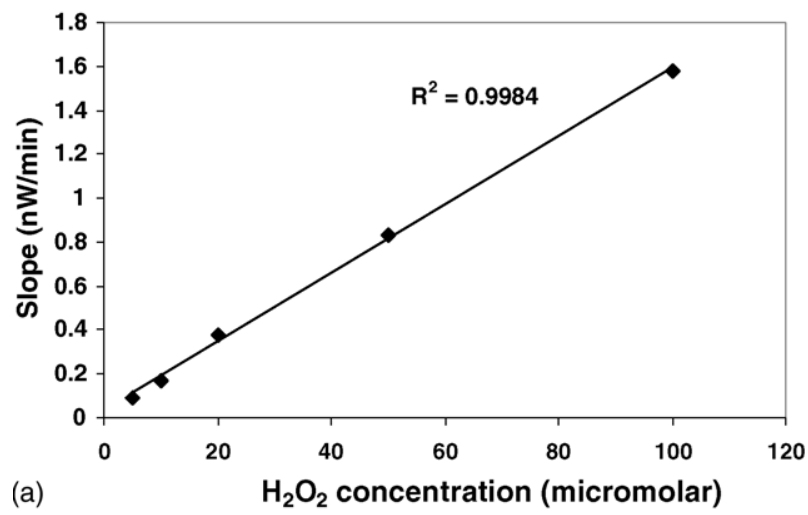

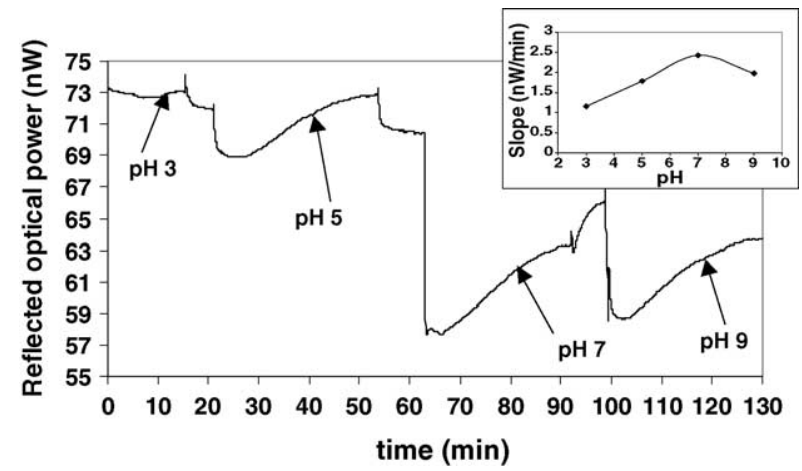

Fig. 5. Reflected optical power for $10 \mu \mathrm{M}$ concentration of $\mathrm{H}_{2} \mathrm{O}_{2}$ at $\mathrm{pH} 3$, 5,7 and 9. Upper right corner: slope of the change of signal for the four $\mathrm{pHs}$ analyzed.

the response is based on the change of signal level. In fact, in the range between pHs 6.5 and 7.5 the dependence is very low, which includes the physiological region.

\subsection{Sensors with capping bilayers}

It has been checked that for sensors of the type of previous section, there is a lost of Prussian Blue during the first measures due to the presence of a buffer solution. This effect has been demonstrated in [25] for Methylene Blue. If the sensor is introduced in a solution at a $\mathrm{pH}$ where binds between polymers and the pigment are broken, Prussian Blue leaves the structure. This leads to a lost of sensitivity.

Two strategies against this phenomenon are suggested in $[25,26]$. In both of them, some capping bilayers are added at the end of the structure. Following the strategy of [25], $\left[\mathrm{PAH}^{+} / \mathrm{PAA}^{-}\right]$bilayers at a pHs between 6 and 6.5 are added because they present a high ionical cross-link in comparison with lower pHs. In this way, they act as a barrier to avoid the leakage of pigment. The first design consists of 17 bilayers of $\left[\mathrm{PAH}+\mathrm{PB}^{+} / \mathrm{PAA}^{-}\right]$at $\mathrm{pH} 5$ and capping bilayers of $\left[\mathrm{PAH}^{+} / \mathrm{PAA}^{-}\right]$at $\mathrm{pH}$ 6. Three sensors have been analyzed in Fig. 6: one of them without capping bilayers, and the other two with two and six bilayers, respectively. If a

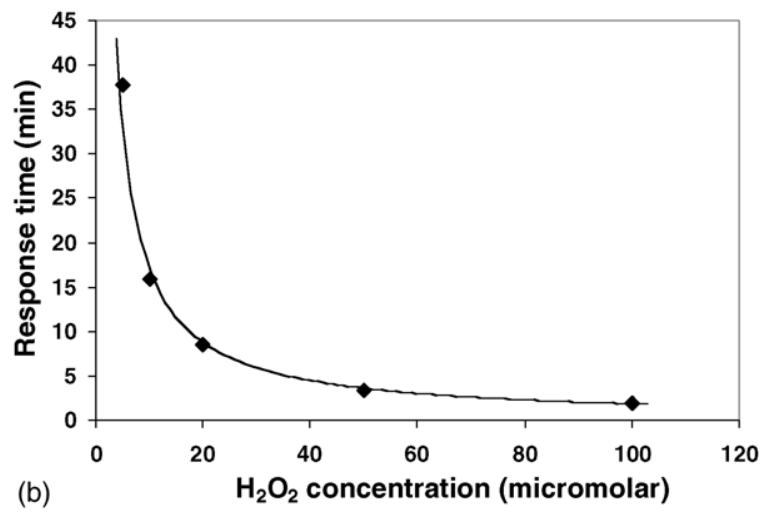

Fig. 4. (a) (Left) slope of the change in the reflected optical power for different concentrations of $\mathrm{H}_{2} \mathrm{O}_{2}$ at $\mathrm{pH}$ 4. Linear tendency line. (b) (Right) response time of the change in the reflected optical power for different concentrations of $\mathrm{H}_{2} \mathrm{O}_{2}$ at $\mathrm{pH}$ 4. Exponential tendency line. 


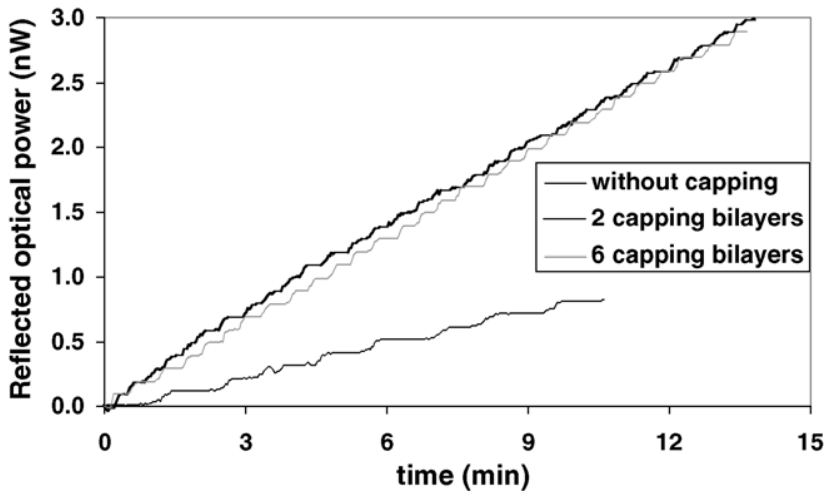

Fig. 6. Comparison of the change in the reflected optical power for three sensors with $\left[\mathrm{PAH}+\mathrm{PB}^{+} / \mathrm{PAA}^{-}\right]_{17}$ at $\mathrm{pH}$ 5. One of them has no capping bilayers and the rest two and six of $\left[\mathrm{PAH}^{+} / \mathrm{PAA}^{-}\right]$at $\mathrm{pH}$, respectively. Measures have been taken at $\mathrm{pH} 7$ and the concentration of $\mathrm{H}_{2} \mathrm{O}_{2}$ is $10 \mu \mathrm{M}$.

$10 \mu \mathrm{M}$ concentration is analyzed at $\mathrm{pH} 7$, both sensors with capping bilayers present a better response. The capping bilayers present no Prussian Blue, so the response should be equal. But, the barrier of $\left[\mathrm{PAH}^{+} / \mathrm{PAA}^{-}\right]$bilayers at $\mathrm{pH} 6$ provokes that Prussian Blue cannot leave the structure as easily. A second experiment (see Fig. 7) where the $\mathrm{pH}$ analyzed is again 7 and the concentration of $\mathrm{H}_{2} \mathrm{O}_{2} 20 \mu \mathrm{M}$, consists of the same structure with the exception that in the first zone a $\mathrm{pH}$ 4.5 has been applied instead of a $\mathrm{pH} 5$, and in the capping bilayers the $\mathrm{pH}$ was 6.5 instead of 6 . The aim was to obtain a higher contrast of ionical strength between the two groups of bilayers, and in this way a better sensitivity. But, results obtained are very similar to those of Fig. 6 . A small change in $\mathrm{pH}$ is sufficient to guarantee that the capping bilayers act as a barrier to avoid the leakage of Prussian Blue. This is corroborated in [27], where a fast variation of the ionical strength at $\mathrm{pH} 6$ is detected for the combination of PAH and PAA. The other conclusion in this first design is that the number of capping bilayers plays no important role in the improvement of the sensitivity of the sensor. This is confirmed in the next strategy analyzed.

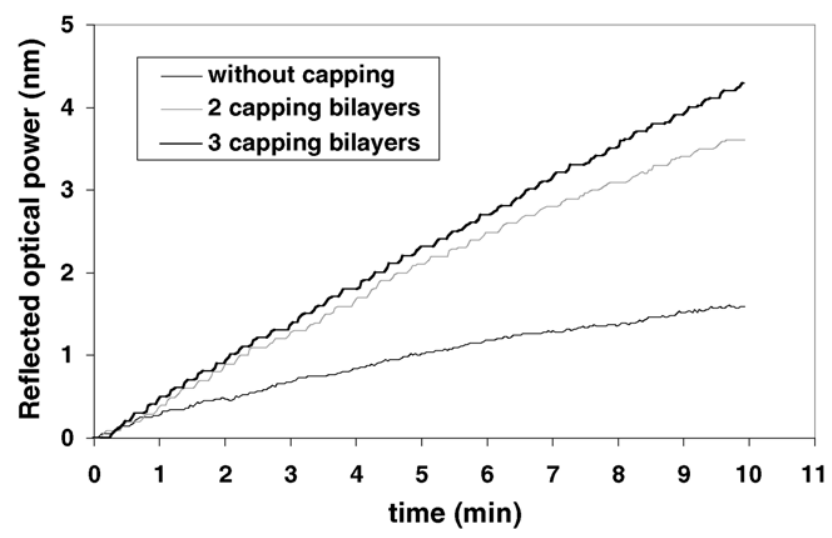

Fig. 7. Comparison of the change in the reflected optical power for three sensors with $\left[\mathrm{PAH}+\mathrm{PB}^{+} / \mathrm{PAA}^{-}\right]_{17}$ at $\mathrm{pH} 4.5$. One of them has no capping bilayers and the rest two and three of $\left[\mathrm{PAH}^{+} / \mathrm{PAA}^{-}\right]$at $\mathrm{pH} 6.5$, respectively. Measures have been taken at $\mathrm{pH} 7$ and the concentration of $\mathrm{H}_{2} \mathrm{O}_{2}$ is $20 \mu \mathrm{M}$.
A second design consists of including a low $\mathrm{pH}$ zone between two high $\mathrm{pH}$ zones. In this way, two barriers avoid the loss of pigment. In the first zone 10 bilayers of the type $\left[\mathrm{PAH}+\mathrm{PB}^{+} / \mathrm{PAA}^{-}\right]$are deposited at a pH 6.5 , in the second zone the same type of bilayers is deposited at a $\mathrm{pH} 4.5$, and finally some capping bilayers are deposited with the same structure as the first zone and $\mathrm{pH}$ 4.5. There is a better response for the sensors where capping bilayers have been added at the end. This effect can be appreciated in Fig. 8a-c,
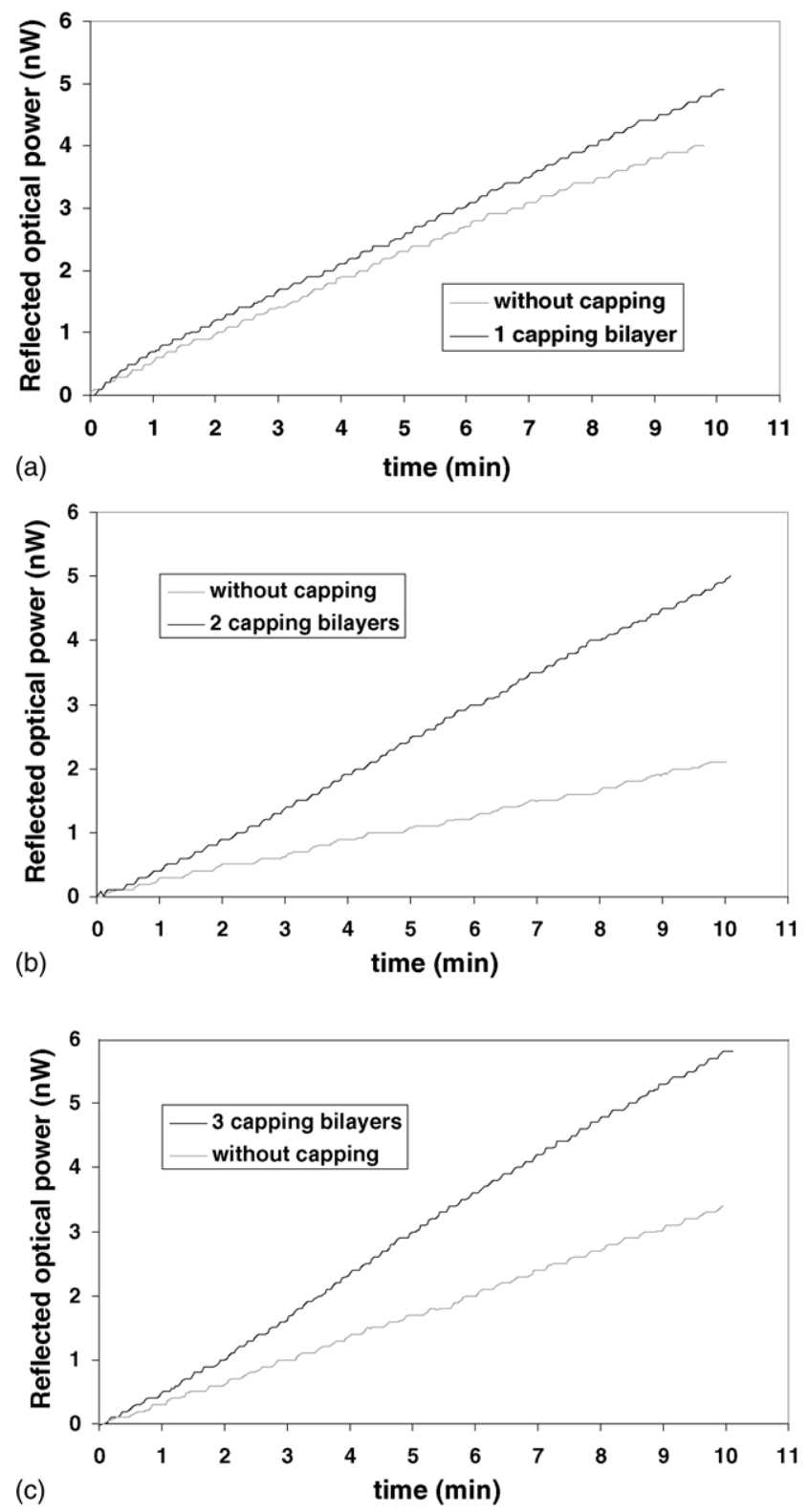

Fig. 8. Comparison of the change in the reflected optical power for six sensors with $\left[\mathrm{PAH}+\mathrm{PB}^{+} / \mathrm{PAA}^{-}\right]_{10}$ at $\mathrm{pH} 6.5$ and $\left[\mathrm{PAH}+\mathrm{PB}^{+} / \mathrm{PAA}^{-}\right]_{7}$ at $\mathrm{pH}$ 4.5. (a) One of them has no capping bilayers and the other one has one capping bilayers of $\left[\mathrm{PAH}+\mathrm{PB}^{+} / \mathrm{PAA}^{-}\right]$at $\mathrm{pH}$ 6.5. (b) One of them has no capping bilayers and the other one has two capping bilayers of $\left[\mathrm{PAH}+\mathrm{PB}^{+} / \mathrm{PAA}^{-}\right]$ at $\mathrm{pH}$ 6.5. (c) One of them has no capping bilayers and the other one has three capping bilayers of $\left[\mathrm{PAH}+\mathrm{PB}^{+} / \mathrm{PAA}^{-}\right]$at $\mathrm{pH} 6.5$. 


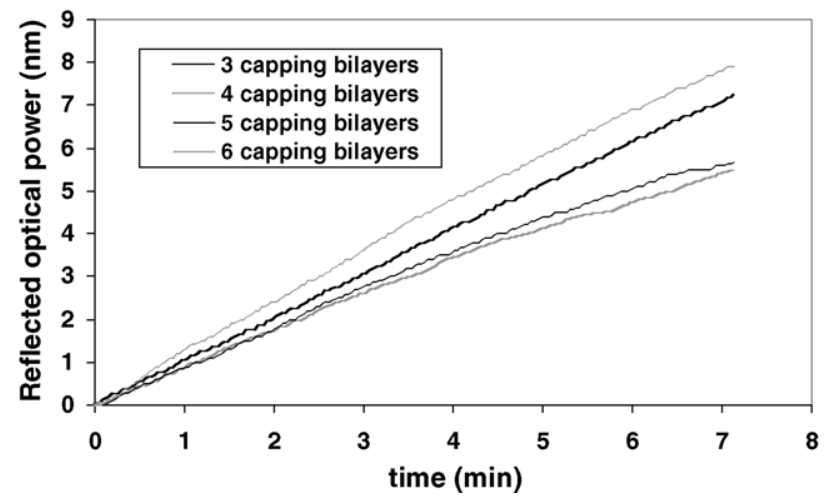

Fig. 9. Comparison of the change in the reflected optical power for four sensors with $\left[\mathrm{PAH}+\mathrm{PB}^{+} / \mathrm{PAA}^{-}\right]_{10}$ at $\mathrm{pH} 6.5$ and $\left[\mathrm{PAH}+\mathrm{PB}^{+} / \mathrm{PAA}^{-}\right]_{7}$ at $\mathrm{pH}$ 4.5. They have respectively $3,4,5$ and 6 capping bilayers of $\left[\mathrm{PAH}+\mathrm{PB}^{+} / \mathrm{PAA}^{-}\right]$at $\mathrm{pH} 6.5$.

where sensors with one, two and three capping bilayers are compared with sensors without capping bilayers that have been immersed the same time in buffer solutions. The $\mathrm{pH}$ analyzed is 7, and the concentration of $\mathrm{H}_{2} \mathrm{O}_{2}$ is $10 \mu \mathrm{M}$. If only one bilayer is deposited, the improvement of the response is not so important, whereas for both two and three capping bilayers there is an appreciable better response. This effect is logical because with one bilayer Prussian Blue can easily escape due to the spread of each individual layer [22]. Regarding the improvement in the overall response of this group of sensors compared with previous ones, this is obvious because more bilayers with colorant have been included. It is remarkable that the i.e. slope of the change of reflected optical power is different between Fig. 8a-c. The reason is that each pair of sensors was immersed a different time in buffer solution. As a result, the response of each pair is degraded in a different manner.

Finally, the same strategy as that of Fig. 8 has been used in Fig. 9. In this case, sensors with 3, 4, 5 and 6 capping bilayers have been constructed in order to analyze the influence of the number of capping bilayers in the improvement of the response of the sensor. The previous assumption that for more than one bilayer there is not a clear influence of the number of stacked bilayers is reaffirmed by the results. Differences between these sensors analyzed are caused by other factors.

\subsection{Interferences}

A wide group of interference compounds have been analyzed. Chloride, bromide, iodide, oxalate, thiocyanate, tartrate, urea, sulfate, phosphate and glucose produce no changes if $2.5 \mathrm{mM}$ concentrations of them are injected in the solutions. Only arsenite $(2.5 \mathrm{mM})$ produced a slight change of the signal but not in the state of Prussian Blue. This is true because the sensor recovered its signal after immersion in another solution without the need of an oxidizing or reducing agent.

\section{Conclusions}

An optical fiber sensor for detection of hydrogen peroxide $\mathrm{H}_{2} \mathrm{O}_{2}$ based on electrostatic self-assembly (ESA) method has been fabricated. The polycation is composed of PAH and Prussian Blue, whereas PAA is used for the polyanion. A simple reflection scheme with a LED source and a photodetector in the first optical communications window serves for detecting changes caused by the presence of $\mathrm{H}_{2} \mathrm{O}_{2}$. The measuring technique is based on the slope of the change of signal produced by a specific concentration of $\mathrm{H}_{2} \mathrm{O}_{2}$. The dependence of the slope of the reflected optical power change as a function of the concentration of $\mathrm{H}_{2} \mathrm{O}_{2}$ is linear in the zone analyzed, whereas the response time as a function of the concentration shows an exponential tendency. The concentrations with a linear response range between $5 \mu \mathrm{M}$ and $0.1 \mathrm{mM}$. A wide range of $\mathrm{pHs}$ is covered: from 3 to 9 , with a low $\mathrm{pH}$ dependent zone located between pHs 6.5 and 7.5.

Some designs have been proposed to avoid the lost of indicator by adding some capping bilayers. Results show an improvement in the response of the sensors if more than one capping bilayer is added to the main structure of the sensor. Adding more bilayers does not lead to an appreciable improvement of the response.

Finally, the sensor has been tested against many interferants showing no cross sensitivity except for ascorbic acid, which is actually the reductive agent.

\section{Acknowledgements}

This work was supported by Spanish Ministerio de Ciencia y Tecnologia and FEDER Research Grants CICYT-TIC 2003-00909, Gobierno de Navarra and FPU MECD Grant. We thank Miguel Valencia and Leire Zabalza of the Public University of Navarra, Spain, for their useful comments, encouragement, and work.

\section{References}

[1] International Standards Organisation, Hydrogen peroxide for industrial use-determination of hydrogen peroxide content-titrimetric method, ISO/DIS 7157 (1996).

[2] H. Liu, Z. Zhang, Y. Fan, Reagentless amperometric biosensor highly sensitive to hydrogen peroxide based on the incorporation of Meldola Blue, fumed-silica and horseradish peroxidase into carbon paste, Fresenius Anal. Chem. 357 (1997) 297.

[3] A. Karyakin, E.E. Karyakina, L. Gorton, The electrocatalytic activity of Prussian Blue in hydrogen peroxide reduction studied using a wall-jacket electrode with continuous flow, J. Electroanal. Chem. 456 (1998) 97.

[4] C. Sun, W. Li, Y. Sun, X. Zhang, J. Shen, Fabrication of multilayer films containing horseradish application as a hydrogen peroxide sensor, Electrochim. Acta 44 (1999) 3401.

[5] J. Zhang, B. Li, Z. Wang, G. Cheng, S. Dong, Functionalized inorganic-organic composite material derivated by sol-gel for construction of mediated amperometric hydrogen peroxide biosensor, Anal. Chim. Acta 388 (1999) 71. 
[6] W. Li, Z. Wang, C. Sun, M. Xian, M. Zhao, Fabrication of multilayer films containing horseradish peroxidase and polication-bearing Os complex by means of electrostatic layer-by-layer absorption and its application as an hydrogen peroxide sensor, Anal. Chim. Acta 418 (2000) 225.

[7] B. Wang, S. Dong, M. Schneider, Sol-gel-derived amperometric biosensor for hydrogen peroxide based on methylene green incorporated in Nafion film, Talanta 51 (2000) 565.

[8] M.P. O'Halloran, M. Pravda, G.G. Guilbault, Prussian Blue bulk modified screen-printed electrodes for $\mathrm{H}_{2} \mathrm{O}_{2}$ detection and for biosensors, Talanta 55 (2001) 605.

[9] L.V. Lukachova, E.A. Kotel'nikova, D. D'Ottavi, E.A. Shkerin, E. Karyakina, D. Moscone, G. Palleschi, C. Antonella, A.A. Karyakin, Nonconducting polymers on Prussian Blue modified electrodes: improvement of selectivity and stability of the advanced $\mathrm{H}_{2} \mathrm{O}_{2}$ transducer, IEEE Sens. J. 3 (2003) 326.

[10] R. Koncki, Chemical sensors and biosensors based on Prussian Blues, Crit. Rev. Anal. Chem. 32 (2002) 79.

[11] B. Li, Z. Zhang, L. Zhao, Chemiluminescent flow-through sensor for hydrogen peroxide based on sol-gel immobilized hemoglobin as catalyst, Anal. Chim. Acta 445 (2001) 161.

[12] H.S. Voraberger, W. Trettnak, V. Ribitsch, Optochemical hydrogen peroxide sensor based on oxygen detection, Sens. Actuators B 90 (2003) 324.

[13] P.S. Grant, M.J. McShane, Development of multilayer fluorescent thin film chemical sensors using Electrostatic Self-Assembly, IEEE Sens. J. 3 (2003) 139.

[14] C. Xu, Z. Zhang, Fluorescence determination of hydrogen peroxide using hemoglobin as mimetic enzyme of peroxidase, Anal. Sci. 17 (1999) 1449

[15] Q. Chen, D. Li, Q. Zhu, H. Zheng, J. Xu, Application of irontetrasulfonatophthalocyanine as a new mimetic peroxidase in the determination of hydrogen peroxide with p-hydroxyphenylpropionic acid as a substrate, Anal. Chim. Acta 381 (1999) 175.

[16] X. Chen, D. Li, H. Yang, Q. Zhu, H. Zheng, J. Xu, Study of tetrasubstituted amino aluminum phthalocyanine as a new red-region substrate for the fluorometric determination of peroxidase and hydrogen peroxide, Anal. Chim. Acta 434 (2001) 51.

[17] F.J. Arregui, Y. Liu, I.R. Matías, R.O. Claus, Optical fiber humidity sensor using a nano Fabry-Perot cavity formed by the ionic selfassembly method, Sens. Actuators B 59 (1999) 54.

[18] Z. Guo, H. Shen, L. Li, Spectrophotometric determination of hydrogen peroxide and glucose based on hemin peroxidase-like catalyzed oxidation of Bromopyrogallol Red, Mikrochim. Acta 131 (1999) 171.

[19] A. Lobnik, M. Cajlakovic, Sol-gel based optical sensor for continuous determination of dissolved hydrogen peroxide, Sens. Actuators B 74 (2001) 194.

[20] R. Koncki, T. Lenarczuk, S. Glab, Optical sensing schemes for Prussian Blue/Prussian White film system, Anal. Chim. Acta 424 (2000) 27.

[21] T. Lenarczuk, D. Wencel, S. Glab, R. Konscki, Prussian Blue-based optical glucose biosensor in flow-injection analysis, Anal. Chim. Acta 447 (2001) 23-32.

[22] G. Decher, Fuzzy nanoassemblies: toward layered polymeric multicomposites, Science 277 (1997) 1232.

[23] F.J. Arregui, I. Latasa, I.R. Matias, R.O. Claus, An optical fiber pH sensor based on the electrostatic self-assembly method, Proceedings of the Second IEEE Sensors Conference, 2003.

[24] F.J. Arregui, Y. Liu, M. Kristie, I.R. Lenahan, R.O. Matías, Claus Optical fiber nanometer-scale Fabry-Perot interferometer formed by the ionic self-assembly monolayer process, Opt. Lett. 24 (1999) 596.
[25] A.J. Chung, M.F. Rubner, Methods of loading and releasing low molecular weight cationic molecules in weak polyectrolyte multilayer films, Langmuir 18 (2002) 1176.

[26] T.C. Wang, Polyelectrolyte Multilayers as Nanostructured Templates for Inorganic Synthesis, Ph.D. Thesis, Dept. Chemical Engineering, Massachusetts Institute of Technology, 2002.

[27] S.S. Shiratori, M.F. Rubner, pH-Dependent thickness behavior of sequentially adsorbed layers of weak polyelectrolytes, Macromolecules 33 (2000) 4213.

\section{Biographies}

Ignacio Del Villar received his MS degree in Electrical and Electronic Engineering in 2002 from the Public University of Navarra (UPNA) and is pursuing the $\mathrm{PhD}$ in the Electrical and Electronic Engineering Department of the Public University of Navarra. His research interest includes optical fiber sensors and the analysis of photonic band-gap structures.

Ignacio R. Matias received his MS degree in Electrical and Electronic Engineering and his $\mathrm{PhD}$ degree, speciality in Optical Fiber Sensors in 1992 and 1996, respectively, from the Polytechnic University of Madrid (UPM), Spain. In 1996, he took up a lectureship at the Public University of Navarra (Pamplona, Spain) where presently he is a permanent Professor. He has co-authored more than 100 journal and conference papers related to optical fiber sensors, passive optical devices and systems. He is an IEEE member. He is an Associate Editor of the IEEE Sensors Journal.

Francisco J. Arregui received the MS degree in electrical engineering from the Catholic University of Navarra, San Sebastian, Spain, in 1994 and the $\mathrm{PhD}$ degree from the Public University of Navarra, Pamplona, Spain in 2000. He has been a member of the CEIT Research Center, San Sebastian, Spain, for 2 years and has been involved in different projects with industry including medical instrumentation, monitoring of high power lines and communications hardware. Since 1995, he has been working at the Public University of Navarra, (Pamplona, Spain). During 1998 and 2000, he was a visiting scientist at the Fiber \& Electro Optics Research Center, Virginia Polytechnic Institute and State University, (Blacksburg, VA, USA). His main research interests include optical fiber sensors, sensor materials and nanostructured materials. He has served as a referee for the journals Optical Engineering, Sensors \& Actuators A, Optics Communications, IEEE Photonics Technology Letters and IEEE Sensors Journal. Francisco J. Arregui is a member of SPIE.

Jesus Echeverria received his MS degree in Advanced Analytical Chemistry from the Bristol University (UK) in 1988, and his $\mathrm{PhD}$ from the Public University of Navarra in 1995. He has been teaching in the Department of Applied Chemistry of the Public University since 1992, in 1998 he was appointed Senior Lecturer. His research interests include adsorption of metals by soils and related pure components and preparation of porous materials.

Richard O. Claus received degrees in engineering from the Johns Hopkins University in the 1970s. Since 1977, he has served on the faculty at Virginia Tech where he is currently the Hester Chair of Engineering and the director of the Fiber \& Electro-Optics Research Center. Claus is a fellow of the IOP and SPIE and has received awards for the application of optical fiber sensors for materials analysis from the ASCE, ASME, IEEE, OSA and ISA. His current interests are optical fiber sensors and self-assembled materials and structures. 